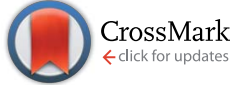

Cite this: J. Mater. Chem. A, 2014, 2 , 19670

Received 14th September 2014 Accepted 7th October 2014

DOI: $10.1039 / \mathrm{c} 4 \mathrm{ta0} 4818 \mathrm{k}$

www.rsc.org/MaterialsA

\section{New dry carbon nanotube coating of over-lithiated layered oxide cathode for lithium ion batteries $\uparrow$}

\begin{abstract}
Junyoung Mun, ${ }^{\text {ab }}$ Jin-Hwan Park, ${ }^{a}$ Wonchang Choi, ${ }^{c}$ Anass Benayad, ${ }^{a}$ Jun-Ho Park, ${ }^{a}$ Jae-Myung Lee, ${ }^{a}$ Seok-Gwang Doo ${ }^{a}$ and Seung M. Oh*d

Carbon serves as one of the best coating materials for the cathode in lithium ion batteries. This is because it can solve two main problems, which are surface deterioration and poor electrical conductivity. However, the conventional carbon coating procedures and, chemical carbonization processes, are especially difficult to implement for the oxide cathode, which could thereby deteriorate the oxide structure. We prepared a new dry $100 \mathrm{~nm}$-thick homogeneous multi-walled carbon nanotube (MWCNT) coating on the high-capacity oxide cathode material, $\mathrm{Li}_{1.17} \mathrm{Ni}_{0.17} \mathrm{Co}_{0.1} \mathrm{Mn}_{0.56} \mathrm{O}_{2}$, by applying shear stress without breaking down the crystal structure or morphology of the cathode. The electronic conductivity of the carbon composite with the coated sample is $170 \mathrm{mS} \mathrm{cm}{ }^{-1}$, which is over 40 times as much as the conductivity of the pristine cathode containing the same amount of carbon. In addition, at a high current condition of $2450 \mathrm{~mA} \mathrm{~g}^{-1}$, a specific capacity of $103 \mathrm{~mA} \mathrm{~h} \mathrm{~g}{ }^{-1}$ is observed even with 3 percent of the carbon (in weight) constituting the coated MWCNT. The unconventionally improved performances are explained by the suppression of the electronic resistance and surface charge transfer resistance by electrochemical analyses.
\end{abstract}

\section{Introduction}

In general, surface deterioration, including the breakdown of surface crystal structure and formation of highly resistive passivation film, caused by the electrochemical side reaction between the electrode and electrolyte, ${ }^{1-4}$ is one of the main concerns in the case of the lithium ion batteries (LIBs). This phenomenon is inevitable because the voltage between the positive and negative electrodes is beyond the electrochemical stability window of the electrolyte to obtain high energy and power density. In this regard, surface coatings have been investigated for their ability to improve the stability of the surface of the electrode and to reduce the failure modes on the interface between the electrode and the electrolyte. Several studies have been proposed to address this issue, but most of them have focused on the inorganic material coating, such as the metal oxides and metal fluorides, because they are highly

${ }^{a}$ Energy Laboratory, Samsung Advanced Institute of Technology, Samsung Electronics
Co. Ltd., 130 Samsung-ro, Suwon, Gyeonggi-do 443-803, Republic of Korea
${ }^{b}$ Department of Energy and Chemical Engineering, Incheon National University, 119
Academy-ro, Songdo-dong, Yeonsu-gu, Incheon 406-772, Republic of Korea
${ }^{c}$ Center for Energy Convergence, KIST, Hwarangno 14gil 5 Seongbuk-gu 136-791,
Republic of Korea
${ }^{d}$ Department of Chemical and Biological Engineering, Seoul National University, 599
Gwanangno, Gwanak-gu, Seoul 151-744, Republic of Korea. E-mail: seungoh@snu.
ac.kr $\dagger$ Electronic supplementary information (ESI) available. See DOI: $10.1039 / \mathrm{c} 4 \mathrm{ta} 04818 \mathrm{k}$ stable. However, they could increase the internal resistivity in the LIB because they are electrically non-conductive materials. ${ }^{5-7}$ In this regard, carbon is considered to be one of the best coating materials. It is highly conductive, electrochemically stable, abundant, environmentally friendly, inexpensive, and it can be easily prepared. Kinetic hindrances and irreversible side reactions can be relieved by improving the electronic conductivity and by passivating the active site on the electrode material to the electrolyte via introducing the carbon coating. Therefore, a great deal of research has been carried out into the carbon coating of lithium ion battery electrodes in order to solve the above mentioned problems. Carbon coatings have been applied to the active materials of LIBs via chemical methods, including the in situ growth of the carbon coating during the active material synthesis and during post-synthetic processes such as chemical vapor deposition or carbon sputtering. ${ }^{8,9}$ Several allotropes of carbons, such as graphite and carbon nanotubes exhibit repeated covalent bonding and high electronic conductivity. Therefore, these conductive carbons are better suited for surface coating. However, the preparations of those kinds of carbons are limited because a high temperature synthesis or catalysis is essential in order to obtain carbon with a particular stacking order or crystallinity. Especially, the coatings of the conductive carbon on the oxide cathode have been very restricted because of the thermal side reactions between the carbon and the oxide at elevated temperature, the so called carbon thermal reduction. To date, carbon coatings on the positive electrode materials of LIBs have been implemented for 
the polyanion-type materials such as $\mathrm{LiFePO}_{4}$ and $\mathrm{LiCoPO}_{4}$. For the oxide cathode materials, only few kinds of carbon coatings have been introduced, using the carbon sputtering, ball milling and co-precipitation methods. ${ }^{\mathbf{8 1 0 - 1 2}}$ However, the number of available methods are limited, owing to the fact that the carbon coating needs to be thin, homogeneous and highly conductive. Additionally, the cathode morphology needs to be preserved after the coating procedure. ${ }^{13-15}$

In an effort to increase the energy density of LIBs and to enhance the use of LIBs in electric vehicles and smart grids, several positive electrode materials showing a high capacity have been explored. In this regard, one of the prominent positive electrode materials is the over-lithiated layered oxide (OLO, $\mathrm{Li}_{x} \mathrm{Mn}_{y} \mathrm{Co}_{z} \mathrm{Ni}_{a} \mathrm{O}_{2}, x>1.0$ ), which exhibits a larger specific capacity than those of $\mathrm{LiCoO}_{2}$ and $\mathrm{LiMn}_{2} \mathrm{O}_{4}$, because of the additional lithium ions in the transition metal site. ${ }^{\mathbf{1 6 - 2 0}}$ Nonetheless, this material is not used in practical LIBs owing to critical drawbacks. Firstly, its electronic conductivity $\left(10^{-9} \mathrm{~S}\right.$ $\mathrm{cm}^{-1}$ ) is poorer than that for the conventional positive electrode materials (for instance, $>10^{-5} \mathrm{~S} \mathrm{~cm}^{-1}$ for $\mathrm{LiCoO}_{2}$ ), which is due to the presence of an insulating $\mathrm{Li}_{2} \mathrm{MnO}_{3}$ phase. ${ }^{8,9}$ For the practical use of this multi-component oxide material in LIBs, a large amount of conductive carbon (over 10\%, and sometimes over $20 \%$ in weight), is necessary to maintain the optimal electronic conductivity of the electrode..$^{21-23}$ However, such a large quantity of the conducting agent cannot be used for producing practical LIBs, because it causes the energy density of the electrode to fall off. Secondly, electrolyte decomposition and film deposition are problematic, because the commonly used electrolytes are not electrochemically stable under the working voltage of OLO $\left(>4.4 \mathrm{~V} v s\right.$. $\left.\mathrm{Li} / \mathrm{Li}^{+}\right){ }^{24-27}$ Therefore, the OLO surface should be passivated in order to avoid the parasitic reactions. Otherwise, resistive surface films continue to grow and increase the electrode polarization, which eventually leads to capacity fading. ${ }^{28-35}$

This work was motivated by the premises that carbon coating on the OLO surface can solve the two above mentioned problems; poor electronic conductivity and parasitic reactions. Normally, a conductive carbon powder is added as a physical mixture with electrode materials to compensate the poor bulk electronic conductivity of electrode materials. The role of conductive carbon is not to enhance the bulk electronic conductivity of the electrode materials but to minimize the contact resistance by providing the electron transfer channel from current collector to the particles of a given electrode material. Unfortunately, this approach seems not viable for the OLO electrode because a large amount of conductive carbon powder is needed for the OLO electrodes. A larger number of contact points between OLO/OLO particles and OLO/current collector are needed to compensate for the poor bulk electronic conductivity of OLO, which causes a decrease in the volumetric energy density of the LIBs due to the larger volume occupied by the passive component (carbon powder). In contrast, if carbon is added as a coating, the electron transfer channel can be provided with a minimal amount of carbon. Note that the contact area between OLO and coated carbon is greatly enlarged because of an intimate contact between the two components. As a result, the contact resistance of the OLO/OLO particles and the OLO/current collector can be effectively reduced. The second problem pertaining to the growth of film resistance as a result of persistent electrolyte decomposition, can also be mitigated by using carbon coating, because the coated carbon can block the surface sites that are responsible for oxidative electrolytic decomposition. ${ }^{36,37}$

In this work, a novel, highly conducting carbon coating procedure is developed for the oxide positive electrode material, OLO, and carried out at ambient temperature. The operational principle is simple; a controlled shear stress is continuously applied to the mixture of OLO powder and multi-walled carbon nanotubes (MWCNTs) in a specially designed mill. This work demonstrates, for the first time to our knowledge, that carbon nanotubes can be uniformly coated onto OLO surfaces using our prescribed method, and the performances of OLO positive electrode can be greatly improved by solving or at least mitigating the two problems mentioned above.

\section{Experimental}

\section{Material preparation}

$\mathrm{Li}_{1.17} \mathrm{Ni}_{0.17} \mathrm{Co}_{0.1} \mathrm{Mn}_{0.56} \mathrm{O}_{2}$ was prepared by oxalate co-precipitation and heat treatment at $900{ }^{\circ} \mathrm{C}$ for $12 \mathrm{~h}$ in air. ${ }^{28}$ The obtained OLO powder was mixed with MWCNTs (E-nanotec, L-purified MWNT, length: 5-15 $\mu \mathrm{m}$ ) by using a pestle and mortar. A controlled amount of MWCNT $(0.5,1,2,3.5$ and 5 weight\% against the OLO powder) was used for sample preparation. The coating was performed at ambient temperature in a Nobilta mini bowl (Hosokawa, Japan), in which a rotating four-way blade is placed $5 \mathrm{~mm}$ away from the wall. A controlled shear stress was continuously applied to the mixture of MWCNTs and OLO powder within the gap by rotating the blade at $3000 \mathrm{rpm}$. The bowl was placed in such a way that the rotating axes were parallel to the ground. The two components were homogeneously mixed with the above setup, by using the effect of gravity.

Material Characterization. XRD analysis was carried out using an X'pert pro (PANalytical) diffractometer with $\mathrm{Cu} \mathrm{K} \alpha$ radiation (1.54056). The Raman spectra were obtained in backscattering mode using a Renishaw inVia system with an argon laser (514.5 $\mathrm{nm})$. The XPS data were obtained using a Physical Electronics (PHI) (quantum 2000 scanning ESCA microprobe) spectrometer. The photoelectrons were excited with an $\mathrm{Al} \mathrm{K} \alpha(1486.6 \mathrm{eV})$ anode operating at a constant power of $100 \mathrm{~W}(15 \mathrm{kV}$ and $10 \mathrm{~mA})$ with an $\mathrm{X}$-ray spot size of $400 \mu \mathrm{m}$. The electronic conductivity of the electrode was measured using a 4-probe DC method under $20 \mathrm{kN}$ and $90 \mathrm{kV}$ (MCP-PD511, Mitsubishi).

\section{Electrochemical analyses}

For the preparation of the composite electrode, a slurry of the prepared sample, Denka black, and poly(vinylidene fluoride) (weight ratio of $94: 3: 3$ ) was coated onto an aluminum current collector. The total amount of carbon including the MWCNT coated on the OLO and Denka black was controlled to 3 weight $\%$. The electrode was then pressed to enhance the interparticle contact. Coin-type cells for both lithium half cells 
and full cells (2032 size, Hohsen) were assembled with the composite electrode, a separator (Celgard), and a counter electrode. A lithium metal disk was used as the counter electrode for the lithium half cell and a composite electrode of graphite was used for the full cell. Galvanostatic cycling was carried out at $12.25 \mathrm{~mA} \mathrm{~g}^{-1}\left(0.1 \mathrm{C}, 1 \mathrm{C}=245 \mathrm{~mA} \mathrm{~g}^{-1}\right)$ for cycle one, with subsequent cycling at $245 \mathrm{~mA} \mathrm{~g}^{-1}$ over the potential range 2.5$4.6 \mathrm{~V}\left(v s . \mathrm{Li} / \mathrm{Li}^{+}\right.$) for lithium half cells and $2.0-4.55 \mathrm{~V}$ for the full cells (model TOSCAT $3500 \mathrm{U}$ ) at $25^{\circ} \mathrm{C}$.

\section{Results and discussion}

As shown in Fig. S1, $\dagger$ a controlled shear stress was applied between the mixture of MWCNTs and the OLO powder (average secondary particle size $=5 \mu \mathrm{m}$ ) by using a rotating blade, resulting into the coating of the cathode material with the MWCNTs. The top view shows the bowl of the Nobilta mini instrument. A rotating four-way blade was designed such that it was almost touching the wall of the bowl with a $5 \mathrm{~mm}$ gap (Fig. S2a $\dagger$ ). The blade rotated with a pre-determined speed (3000 rpm), and controlled shear stress was continuously given to the surface of the mixture of the OLO powder and the MWCNTs in the prepared $5 \mathrm{~mm}$ gap. After applying the shear stress, the MWCNT coated OLO was obtained because the smaller powder (the MWCNTs) began aggregating the surface of the larger powder (the OLO). Furthermore, the powders were coated homogeneously by leaning the bowl perpendicularly, in such a way that the rotating axis was parallel to the ground, thus mixing the powder by gravity (Fig. S2b $\dagger$ ).
Fig. 1a shows the X-ray diffraction (XRD) pattern of the prepared samples. The MWCNT-coated OLO powder sample gives the diffraction peaks belonging to the pristine OLO without additional peaks, indicative of no change in the OLO crystal structure during the coating process. This must be due to the low-temperature coating process without carbothermal reduction. Moreover, the scanning electron microscopy (SEM) images illustrate that the apparent particle morphology does not change. The secondary OLO particles, which are formed by the aggregation of the nanometer-sized primary particles, are not broken, reflecting that the applied shear stress is not strong enough to damage the secondary particles as well as the primary particles (insets of Fig. 1b and c). Carbon coating can, however, be confirmed by inspecting the high-resolution field-emission scanning electron microscopy (FE-SEM) images. As seen in Fig. 1c and d, the coated OLO particles carry the MWCNTs on their surface. For these samples, the coating of MWCNTs improves as the coating time increases from 3 to 20 minutes. Carbon coating is further confirmed by inspecting the cross sectional images. The surface region of the pristine OLO appears clean without any foreign materials (Fig. 1e). However, the coated sample carries a homogeneous ca. 100 nm-thick MWCNT layer on its surface (Fig. 1f).

Even if the OLO particles used in this study are robust enough to face the applied shear stress, it is likely that the MWCNTs are prone to damage because they have a tube-type morphology and a high aspect ratio that can be broken more easily than the round-shaped OLO particles. This feature is ascertained by Raman analysis that was performed as a function
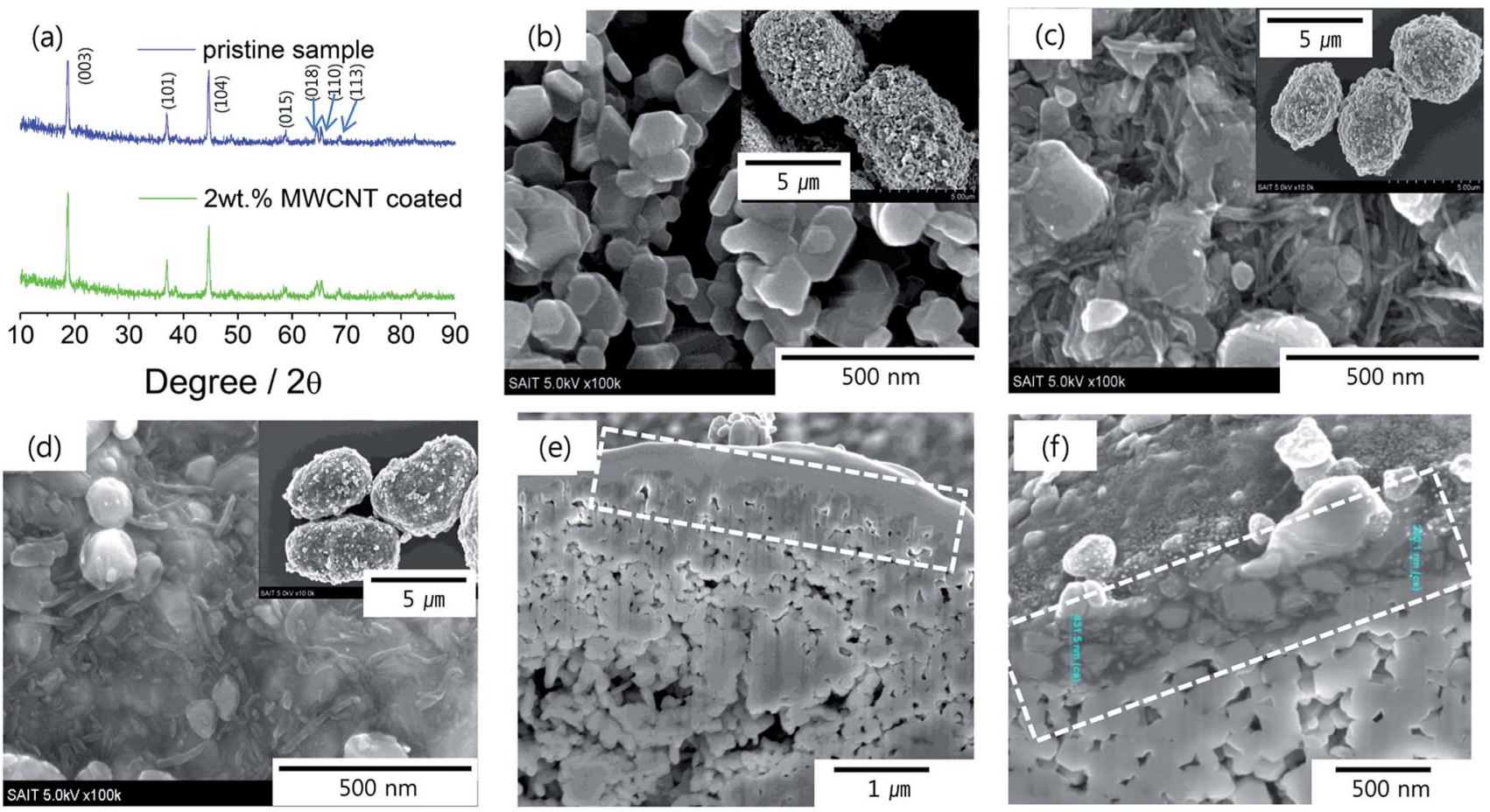

Fig. 1 (a) XRD patterns of the pristine and 2 weight\% MWCNT-coated (coating time $=20$ min) OLO powders. FE-SEM top views of (b) pristine, (c) MWCNT-coated (coating time $=3 \mathrm{~min}$ ) and (d) MWCNT-coated (coating time $=20 \mathrm{~min}$ ). Cross-sectional FE-SEM images of (e) pristine and ( $\mathrm{f}$ ) MWCNT-coated (coating time $=20 \mathrm{~min}$ ) OLO (surface region is highlighted by rectangles with white dotted line). 


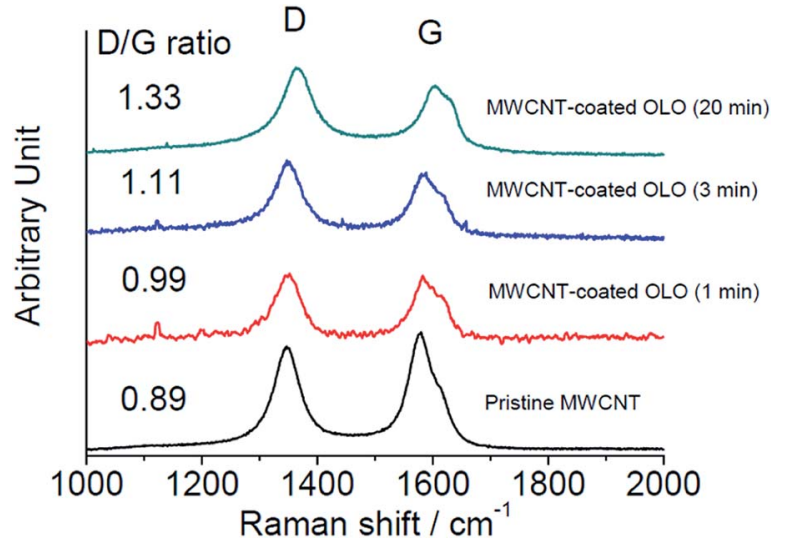

Fig. 2 Raman spectra obtained from the pristine MWCNT and MWCNT-coated OLO samples. The coating time and calculated D/G ratio are indicated in the inset.

of the coating time (Fig. 2). The pristine MWCNTs gave two characteristic Raman peaks that come from the disordered carbon structure (D peak, $1350 \mathrm{~cm}^{-1}$ ) and the ordered graphitic structure (G peak, $\left.1590 \mathrm{~cm}^{-1}\right) .{ }^{38}$ Although it is hard to observe D peak ( $A_{1}^{\prime}$ symmetry vibration of carbons) in the crystal of graphite owing to its perfect symmetry, the number of defects on the MWCNT increases the intensity of the D peak. In contrast, the $\mathrm{G}$ peak ( $E_{2 \mathrm{~g}}$ symmetry vibration of carbons) is generated from the in-plane vibrations of the graphene layers and decreases as the site of defect interferes the vibration. The MWCNTs that suffered from the applied shear stress for a longer period of time show an increase in the $\mathrm{D}$ peak at the expense of the $\mathrm{G}$ peak (from 0.89 to 1.33 in the $\mathrm{D} / \mathrm{G}$ ratio), which must be due to cleavage of MWCNTs and conversion of the ordered graphene structure into the disordered amorphous state.

The coverage of MWCNTs on the OLO surface was assessed by using X-ray photoelectron spectroscopy (XPS) as a function of the coating time. The $\mathrm{C} 1 \mathrm{~s}, \mathrm{O} 1 \mathrm{~s}$, and Mn $2 \mathrm{p}$ XPS spectra are presented in Fig. 3. As seen in the C 1s spectra, the MWCNTcoated OLO sample shows a peak at $285 \mathrm{eV}$, which is assigned to the $\mathrm{C} 1 \mathrm{~s}$ photoelectrons emitted from the $\mathrm{C}-\mathrm{C}$ moiety. ${ }^{17,27,35}$ This peak becomes stronger, indicative of thicker or more uniform coverage of MWCNTs with an increase in the coating time. The MWCNT-coated OLO samples also showed weak peaks at $286 \mathrm{eV}$ (C-O or defects on carbon) and $289 \mathrm{eV}\left(\mathrm{CO}_{3}{ }^{2-}\right) \cdot{ }^{17,27,35}$ This feature suggests that the surface of MWCNTs is partially oxidized or that defects form during the coating process. Thicker or more uniform carbon coverage with an increase in coating time is also confirmed from the $\mathrm{O} 1 \mathrm{~s}$ and $\mathrm{Mn} 2 \mathrm{p}$ spectra. The $\mathrm{O} 1 \mathrm{~s}$ photoelectrons at $529.5 \mathrm{eV}$ and $531.5 \mathrm{eV}$, which are emitted from the oxide lattice in OLO, become less intense with the increasing coating time. ${ }^{17,27,35}$ Similarly, the intensity of the Mn 2 $\mathrm{p}_{3 / 2}(642 \mathrm{eV})$ and $\mathrm{Mn} 2 \mathrm{p}_{1 / 2}(654 \mathrm{eV})$ photoelectrons that are emitted from $\mathrm{Mn}^{4+}$ ions in OLO becomes weaker with an increase in coating time since a thicker or more uniform carbon layer is coated on the OLO surface. In short, a high coverage of MWCNT is achieved by using the simple physical coating method. The relative atomic concentration for each element is summarized in Table $\mathrm{S} 1, \dagger$ in which it is seen that the atomic concentration of carbon amounts to $75.2 \%$ after a $20 \mathrm{~min}$ coating. In short, a high level of MWCNT coverage is achieved by using the simple physical coating method.

In order to confirm the role of coated MWCNT on OLO, which is expectedly the mitigation of contact resistance by providing the electron transfer channel, electronic conductivity was measured for the composite electrodes prepared from the pristine and MWCNT-coated OLO. To simulate the real electrode preparation, a conductive carbon powder (Denka black) was added, but the total amount of carbon (sum of Denka black and MWCNTs) was fixed at the predetermined values for a fair comparison. As shown in Fig. 4, the electrodes prepared from the MWCNT-coated OLO show higher electronic conductivity for every composition. When the carbon loading is high (86:8), the conductivity difference is only marginal $\left(1300 \mathrm{mS} \mathrm{cm}{ }^{-1}\right.$ for the pristine OLO and $1429 \mathrm{mS} \mathrm{cm}^{-1}$ for the MWCNT-coated OLO), probably due to the fact that the Denka black particles fill almost all the void spaces between the OLO particles. However, the conductivity difference becomes substantial with a decrease in the loading of the total carbon. For instance, when the OLO : total carbon ratio is $94: 3$, the electronic conductivity is $170 \mathrm{mS} \mathrm{cm}{ }^{-1}$ for the MWCNT-coated OLO and $4 \mathrm{mS} \mathrm{cm}^{-1}$ for the pristine OLO. In this carbon-depleted composition, the (a) C1s

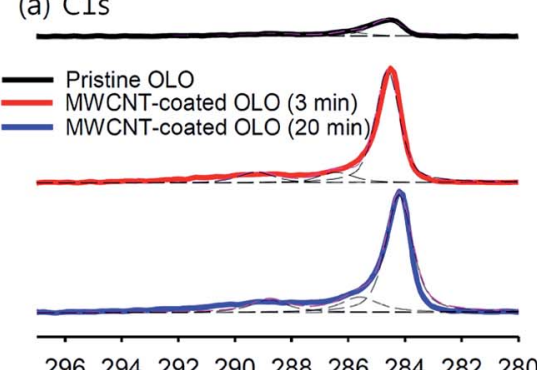

Binding Energy / eV (b) $01 \mathrm{~s}$

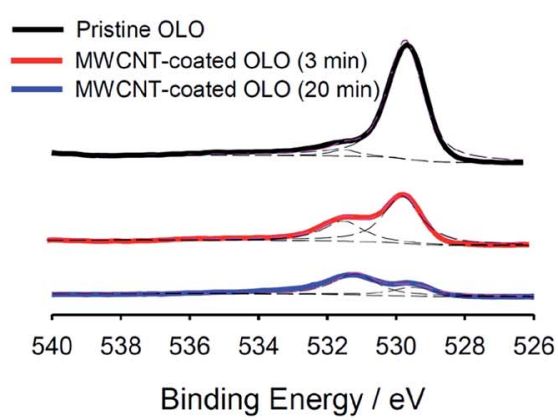

(c) $\mathrm{Mn} 2 \mathrm{p}$

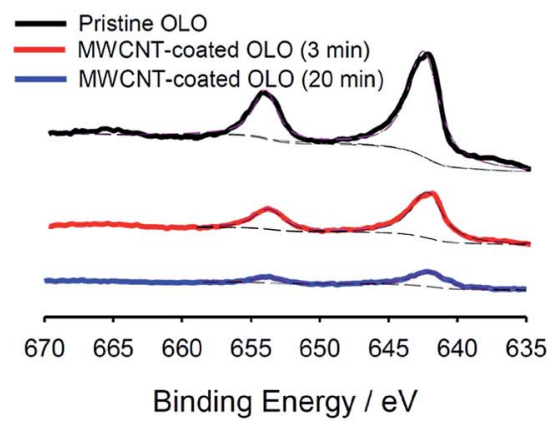

Fig. 3 XPS spectra of (a) C 1s, (b) O 1s, and (c) Mn 2p obtained from the pristine and MWCNT-coated samples. The fitted curves are indicated by the dotted lines. 


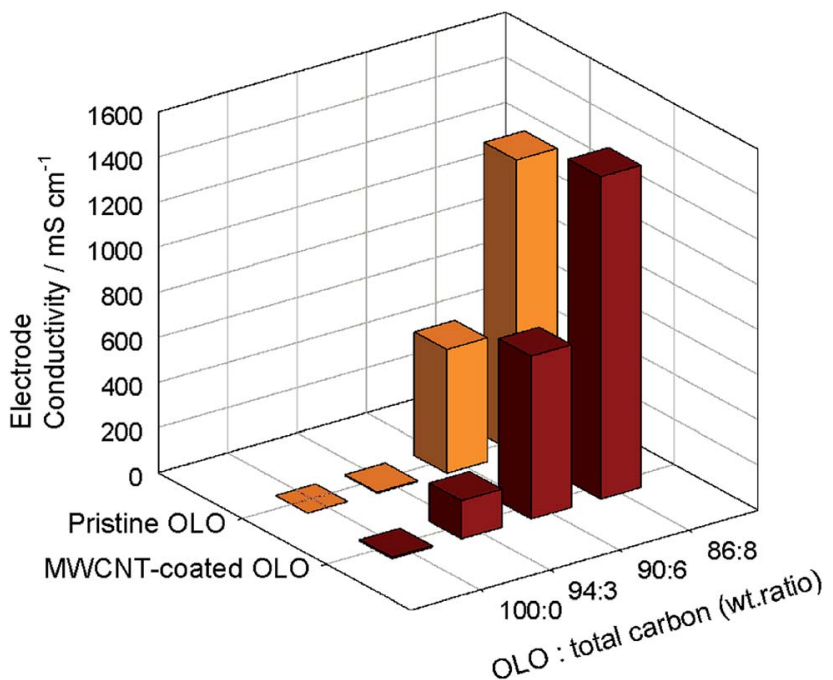

Fig. 4 Electronic conductivity of the composite electrodes prepared from the pristine and MWCNT-coated OLO (coating time $=20 \mathrm{~min}$ ) powders. Total carbon amount including MWCNT and Denka black was controlled to each weight ratio by adding Denka black. contact resistance should be high for the pristine OLO because the contact area between OLO and carbon particles is small. In the case of MWCNT-coated OLO, the contact area is already large enough, such that the high electrode conductivity can be achieved even if the loading of Denka black is low.

Fig. S3† exhibits the initial Coulombic efficiencies and rate capabilities with varying amounts of MWCNT coating. Below a 2 weight $\%$ MWCNT, the initial Coulombic efficiency and rate capability of $2 \mathrm{C} / 0.2 \mathrm{C}$ continuously increased with the increasing amount of carbon. In a previous report, the discharge polarization was found to be high relative to the charge sequence. Furthermore, the $1^{\text {st }}$ Coulombic efficiency can be improved over $90 \%$ by controlling the testing temperatures via room temperature charging and high temperature discharging to lower the kinetic parameter during discharging. ${ }^{6,39}$ It was believed that the enhanced conductivity lowered the polarization during the discharge and increased the discharge capacity, thus improving the initial Coulombic efficiency. However, OLO needs to be charged over $4.4 \mathrm{~V} v s . \mathrm{Li}_{/} \mathrm{Li}^{+}$to enhance the obtained specific capacity by using the lithium ions in $\mathrm{Li}_{2} \mathrm{MnO}_{3}$. Under highly oxidative conditions, the unfavorable
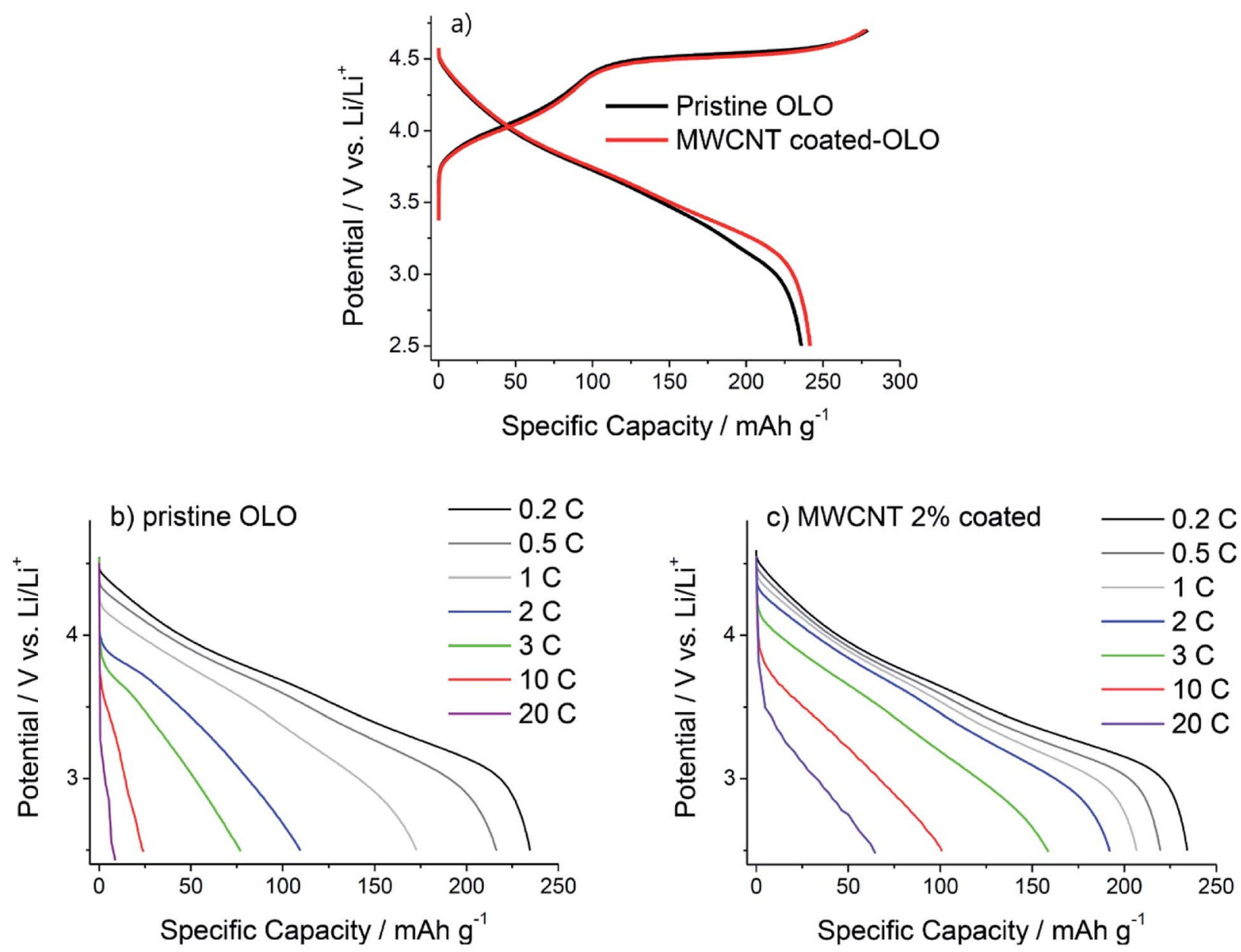

Fig. 5 (a) First voltage profiles obtained from lithium half cells with pristine and 2 weight\% MWCNT-coated OLO. Current density: 2.45 mA g ${ }^{-1}$ $(0.1 \mathrm{C})$; voltage cut-off: $2.5-4.7 \mathrm{~V}\left(\mathrm{vs}\right.$. $\left.\mathrm{Li} / \mathrm{Li}^{+}\right)$; discharge voltage profiles from the same cell with (b) pristine and (c) 2 weight\% MWCNT-coated OLO with various discharge current densities after the controlled charge sequence (current density: 0.5 C; constant voltage mode was added to the end of charge until $0.05 \mathrm{C}$ ). 


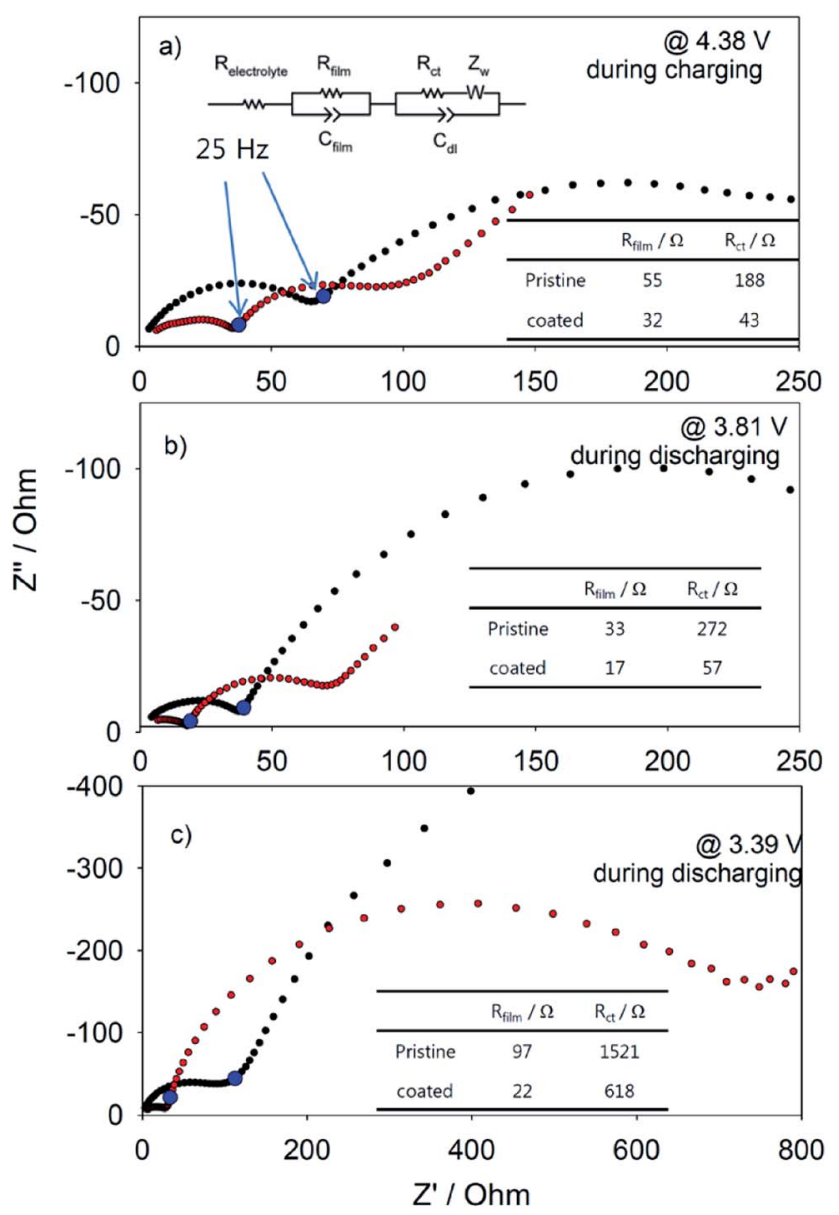

Fig. 6 AC impedance spectra (as a Nyquist) obtained with the 3electrode cell with pristine (black square) and 2 wt\% MWCNT-coated OLO (red square) (a) at the $4.38 \mathrm{~V}$ during the $1^{\text {st }}$ charging, (b) at the 3.81 $\mathrm{V}$ and $(\mathrm{c})$ at the $3.39 \mathrm{~V}$ during the $1^{\text {st }}$ discharge at $25^{\circ} \mathrm{C}$. The proposed equivalent circuit for fitting is depicted on the left upper. Each fitted values of $R_{\mathrm{film}}$ and $R_{\mathrm{ct}}$ are shown. electrolyte side reaction is accelerated on the surface of OLO. From this, it is also predicted that high Coulombic efficiency is originated from the electrochemically stable carbon coating which blocks the unfavorable side reaction of the electrolyte on the surface of OLO. Above the 2 weight $\%$ MWCNT, there was no further improvement; therefore, the amount of carbon was set to this concentration.

Fig. 5a compares the charge/discharge voltage profiles obtained from the Li/pristine OLO and Li/MWCNT-coated OLO cell in the first cycle. The specific capacity for both charging and discharging is comparable for two electrodes. The similarity in the latent capacity in turn illustrates that the OLO crystal structure is not changed during the coating due to the lowtemperature process without carbothermal reduction, in agreement with the XRD results (Fig. 1a). In contrast, the specific discharge capacity for using the lithium ion batteries was higher in the case of the MWCNT-coated sample (bare: 234.8 and MWCNT-coated sample: $241.5 \mathrm{~mA} \mathrm{~h} \mathrm{~g}^{-1}$ ). The MWCNT-coated sample showed a higher discharge capacity, and the improved conductivity relieved the kinetic hindrances that restricted the discharge capacity, especially at the end of discharge under $3.6 \mathrm{~V}$. These changes were related to the lithiation process involving the activated $\mathrm{MnO}_{2}$ derived from $\mathrm{Li}_{2} \mathrm{MnO}_{3}$ during the $1^{\text {st }}$ charge sequence over $4.4 \mathrm{~V}$.

The rate capability is compared for the two electrodes in the discharging sequence in Fig. 5b and c. Clearly, the MWCNTcoated OLO outperforms the pristine OLO. For instance, the discharge capacity obtained at $10 \mathrm{C}\left(2450 \mathrm{~mA} \mathrm{~g}^{-1}\right)$ amounts to $103 \mathrm{~mA} \mathrm{~h} \mathrm{~g}^{-1}$ for the coated electrode, but it is only $24 \mathrm{~mA} \mathrm{~h} \mathrm{~g}^{-1}$ for the pristine electrode. The discharge voltage profiles shown in Fig. $5 b$ and $c$ reveal that the contact resistance of the OLO/ OLO particles has a strong effect on the rate property. The two electrodes show a big difference in the ohmic resistance, which appears as a vertical potential drop at the very early period of the discharge. When the OLO electrodes are discharged at slower rates $(0.2-0.5 \mathrm{C})$, the electrode potential drop is marginal and comparable for the two electrodes. However, when the rate is
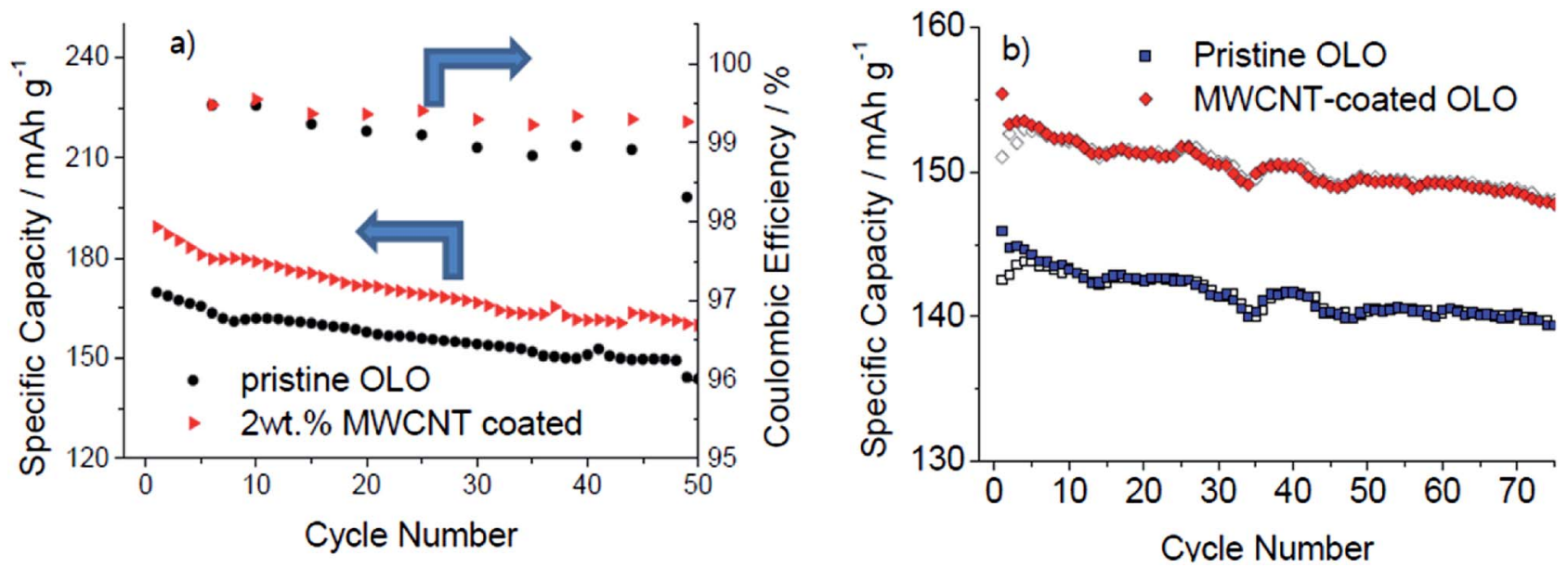

Fig. 7 (a) The obtained cyclability and Coulombic efficiency (average of 5 cycles) from the half cell with pristine and 2 wt\% MWCNT-coated samples; (b) cyclability of the full cell with pristine and 2 weight\% MWCNT-coated sample with constant charge and discharge current (1 C) at ambient temperature. 
increased, the potential drop itself becomes higher and the difference between the potential drops becomes larger for the two electrodes. At $10{ }^{\circ} \mathrm{C}$, for instance, the potential of the pristine OLO electrode drops down to $3.5 \mathrm{~V}$ right after the cell is discharged, whereas the potential of the coated OLO electrode remains at $3.9 \mathrm{~V}$ at the same discharge rate. The shape of the voltage profile as well as the ohmic resistance also get altered by the MWCNT coating. Especially, the plateaus between 3.5 and 3.0 V show significant improvement because of the MWCNT coating. In the case of the pristine sample, the effect of these resistive behaviours becomes more critical under high current condition than that under the moderate current conditions. These severe obstacles are significantly relieved by the MWCNT coating on the OLO surface.

To investigate the resistance behaviour in more detail, AC impedance analysis at various stages of lithiation and delithiation was carried out. The AC impedance results obtained from 3-electrode cells, comprising the prepared electrode as the working electrode and two lithium electrodes as the counter and reference electrodes, exhibit some differences. Two semicircles can be clearly observed in Fig. 6; diameter of the semicircle at high frequency correspond to the resistance of the surface film and that at low frequency correspond to the chargetransfer resistance of lithium reactions occurring on the electrode surface. ${ }^{38}$ The impedance results were fitted with the equivalent circuit shown in Fig. 6a. The most revealing feature in Fig. $6 \mathrm{a}-\mathrm{c}$ is that both the semicircles of the MWCNT-coated OLO are always smaller than those of the pristine OLO, demonstrating that the kinetic hindrances were alleviated. It is speculated that this was due to the enhanced surface stability and electronic conductivity of the electrode owing to the MWCNT-coating. It also improved the kinetic characteristics of charge-transfer resistance by increasing the active sites for the electrochemical reaction without isolating the active material. In the case of the pristine OLO, as lithiation proceeded after the $1^{\text {st }}$ charging, the high frequency-circle in the Nyquist plot obtained during discharging decreased at $3.81 \mathrm{~V}$, but increased again at $3.39 \mathrm{~V}$ on further discharging owing to the insulation caused by the surface film deposition of materials such as $\mathrm{Li}_{2} \mathrm{CO}_{3}$ and $\mathrm{Li}_{2} \mathrm{O}$, from the electrolytic decomposition or oxidation by the oxygen from OLO..$^{37,40}$ The behaviour of the low frequency circle was also similar to that of the high frequency circle. The charge-transfer resistances increased greatly at the end of discharge sequences, corresponding to sluggish delithiation and lithiation reactions owing to surface film. ${ }^{1726}$ The resistance during the discharge was much higher than that during the charging process. Unlike the pristine OLO, the film resistance of the MWCNT-coated OLO during discharge decreased from 32 to $22 \Omega$. The high resistive behaviour of the OLO on the discharging was also greatly relieved by the MWCNT coating. This was in good agreement with the polarization, which was highly reduced during the discharge sequence in the voltage profiles (Fig. 5a) and rate capability test (Fig. 5b and c).

The cyclability was found to be reasonable after coating with MWCNT, with the obtained capacity being $>180 \mathrm{~mA} \mathrm{~h} \mathrm{~g}^{-1}$ even for the $94: 3$ electrode composition. The specific capacity achieved for the MWCNT-coated electrode was higher than that of the pristine sample; the average Coulombic efficiency during 50 cycles ( 2 weight\% of the MWCNT-coated OLO: 99.5\%) was also higher than that for the pristine samples (99.1\%) (Fig. 7a). It is speculated that the surface carbon coating additionally blocks the continuous electrolyte decomposition on the cathode under oxidative conditions at a high potential.

Finally, we fabricated full cells using the prepared cathode samples and a graphite anode. A full cell with the 2 weight\% MWCNT-coated OLO exhibited a higher discharge capacity, exceeding $150 \mathrm{~mA} \mathrm{~h} \mathrm{~g}^{-1}$ (divided by the mass of OLO), as compared to that containing the pristine sample at a constant current/constant voltage rate of $245 \mathrm{~mA} \mathrm{~h} \mathrm{~g}^{-1}$ between 2.5 and $4.6 \mathrm{~V}$, at $25{ }^{\circ} \mathrm{C}$. Importantly, the obtained cyclability was highly stable even after 70 cycles (Fig. 7b).

\section{Conclusions}

The MWCNT coating on the OLO cathode material introduced here is the first system that has been reported to overcome the kinetic hindrances of poor initial Coulombic efficiency and rate capability. We confirmed that the MWCNT coating could be successfully applied by using a simple physical coating method that prevents any breakdowns in the cathode structure. These improvements have great potential for producing improved lithium ion batteries, because the methodology could be used for various different cathode materials, without damaging their original crystal structure. These results pave the way for highly conductive MWCNT coatings on oxide cathode materials.

\section{Acknowledgements}

The acknowledgements come at the end of an article after the conclusions and before the notes and references.

\section{Notes and references}

1 M. Gu, I. Belharouak, J. Zheng, H. Wu, J. Xiao, A. Genc, K. Amine, S. Thevuthasan, D. R. Baer, J. G. Zhang, N. D. Browning, J. Liu and C. Wang, ACS Nano, 2013, 7, 760.

2 G. Jeong, Y.-U. Kim, H. Kim, Y.-J. Kim and H.-J. Sohn, Energy Environ. Sci., 2011, 4, 1986.

3 Y. K. Sun, S. T. Myung, B. C. Park, J. Prakash, I. Belharouak and K. Amine, Nat. Mater., 2009, 8, 320.

4 Y.-S. Kang, T. Yoon, J. Mun, M. S. Park, I.-Y. Song, A. Benayad and S. M. Oh, J. Mater. Chem. A, 2014, 2, 14628.

5 Y.-K. Sun, M.-J. Lee, C. S. Yoon, J. Hassoun, K. Amine and B. Scrosati, Adv. Mater., 2012, 24, 1192.

6 K. J. Rosina, M. Jiang, D. Zeng, E. Salager, A. S. Best and C. P. Grey, J. Mater. Chem., 2012, 22, 20605.

7 W. Choi, A. Benayard, J.-H. Park, J. Park, S.-G. Doo and J. Mun, Electrochim. Acta, 2014, 117, 492.

8 A. Ponrouch, A. R. Goñi, M. T. Sougrati, M. Ati, J. M. Tarascon, J. Nava-Avendaño and M. R. Palacín, Energy Environ. Sci., 2013, 6, 3363.

9 H. Li and H. Zhou, Chem. Commun., 2012, 48, 1201.

10 J. Liu, Q. Y. Wang, B. Reeja-Jayan and A. Manthiram, Electrochem. Commun., 2010, 12, 750-753. 
11 Q. Cao, H. P. Zhang, G. J. Wang, Q. Xia, Y. P. Wu and H. Q. Wu, Electrochem. Commun., 2007, 9, 1228.

12 S. Luo, K. Wang, J. Wang, K. Jiang, Q. Li and S. Fan, Adv. Mater., 2012, 24, 2294-2298.

13 A. Varzi, D. Bresser, J. von Zamory, F. Müller and S. Passerini, Adv. Energy Mater., DOI: 10.1002/aenm.201400054.

14 P. Manikandan, P. Periasamy and R. Jagannathan, J. Mater. Chem. A, 2013, 1, 15397.

15 Y. Zhou, J. Wang, Y. Hu, R. O'Hayre and Z. Shao, Chem. Commun., 2010, 46, 7151.

16 B. Li, H. Yan, J. Ma, P. Yu, D. Xia, W. Huang, W. Chu and Z. Wu, Adv. Funct. Mater., 2014, 24, 5112.

17 N. Yabuuchi, K. Yoshii, S. T. Myung, I. Nakai and S. Komaba, J. Am. Chem. Soc., 2011, 133, 4404.

18 K.-S. Park, D. Im, A. Benayad, A. Dylla, K. J. Stevenson and J. B. Goodenough, Chem. Mater., 2012, 24, 2673.

19 J. Bareno, C. Lei, J. Wen, S. H. Kang, I. Petrov and D. Abraham, Adv. Mater., 2010, 22, 1122.

20 M. G. Kim and J. Cho, Adv. Funct. Mater., 2009, 19, 1497.

21 D. Wang, I. Belharouak, G. Zhou and K. Amine, Adv. Funct. Mater., 2013, 23, 1070.

22 Y. K. Sun, Z. Chen, H. J. Noh, D. J. Lee, H. G. Jung, Y. Ren, S. Wang, C. S. Yoon, S. T. Myung and K. Amine, Nat. Mater., 2012, 11, 942.

23 D. Mohanty, S. Kalnaus, R. A. Meisner, K. J. Rhodes, J. Li, E. A. Payzant, D. L. Wood Iii and C. Daniel, J. Power Sources, 2013, 229, 239.

24 A. Van Bommel and J. R. Dahn, Electrochem. Solid-State Lett., 2010, 13, A62.

25 Z. Li, F. Du, X. Bie, D. Zhang, Y. Cai, X. Cui, C. Wang, G. Chen and Y. Wei, J. Phys. Chem. C, 2010, 114, 22751.
26 M. Jiang, B. Key, Y. S. Meng and C. P. Grey, Chem. Mater., 2009, 21, 2733.

27 S. K. Martha, J. Nanda, G. M. Veith and N. J. Dudney, J. Power Sources, 2012, 216, 179.

28 K.-S. Park, A. Benayad, M.-S. Park, W. Choi and D. Im, Chem. Commun., 2010, 46, 4190.

29 H. K. Song, K. T. Lee, M. G. Kim, L. F. Nazar and J. Cho, Adv. Funct. Mater., 2010, 20, 3818.

30 E. S. Lee and A. Manthiram, J. Electrochem. Soc., 2011, 158, A47.

31 S. H. Kang and M. M. Thackeray, Electrochem. Commun., 2009, 11, 748.

32 B. Liu, Q. Zhang, S. He, Y. Sato, J. Zheng and D. Li, Electrochim. Acta, 2011, 56, 6748.

33 Y. S. Park, K. H. Choi, H. K. Park and S. M. Lee, J. Electrochem. Soc., 2010, 157, A850.

34 Y. S. Jung, P. Lu, A. S. Cavanagh, C. Ban, G.-H. Kim, S.-H. Lee, S. M. George, S. J. Harris and A. C. Dillon, Adv. Energy Mater., 2012, 3, 213.

35 J. Mun, S. Kim, T. Yim, J. H. Ryu, Y. G. Kim and S. M. Oh, J. Electrochem. Soc., 2010, 157, A136.

36 M. Noh, Y. Kwon, H. Lee, J. Cho, Y. Kim and M. G. Kim, Chem. Mater., 2005, 17, 1926.

37 J. Wolfenstine, J. Read and J. Allen, J. Power Sources, 2007, 163, 1070.

38 J. Mun, T. Yim, S. Jurng, J. H. Park, S. Y. Lee, J. H. Ryu, Y. G. Kim and S. M. Oh, Electrochem. Commun., 2011, 13, 1256.

39 B. Qiu, J. Wang, Y. Xia, Z. Wei, S. Han and Z. Liu, J. Power Sources, 2014, 268, 517.

$40 \mathrm{H}$. Yu, Y. Wang, D. Asakura, E. Hosono, T. Zhang and H. Zhou, RSC Adv., 2012, 2, 8797. 